\title{
Combined nerve and tendon transfer to restore elbow extension in tetraplegic patients: surgical technique and case report
}

\author{
Marco Biondi ${ }^{1}$ Maura Zucchini ${ }^{1} \cdot$ Prospero Bigazzi $^{1} \cdot$ Giuseppe Falcone $^{2} \cdot$ Sandra Pfanner $^{1} \cdot$ Giulio Del Popolo $^{2}$
}

Received: 16 December 2019 / Revised: 10 April 2020 / Accepted: 13 April 2020

(C) International Spinal Cord Society 2020

\begin{abstract}
Introduction In individuals with tetraplegia, elbow extension is critical for overhead activities, weight shifting, independent transfers, and to perform self-care tasks such as eating. At present, restoration of elbow extension in tetraplegic patients can be performed using either tendon or nerve transfers. Each procedure presents several advantages and limitations that must be discussed with the potential surgical candidate, based on remaining muscular functions and functional goals.

Case presentation We propose a novel combined technique of both tendon and nerve transfer to restore active elbow extension by transferring the posterior deltoid tendon to the triceps tendon and the branch of teres minor nerve to the long head of the triceps nerve. Techniques were performed from the same shoulder posterior surgical approach.

Discussion This surgical technique can add the benefits of each tendon and nerve transfer, leading to a reduction of failure rates, with more predictable outcomes.
\end{abstract}

\section{Introduction}

At present, there is a debate regarding the optimal technique for restoration of elbow extension in patients with spinal cord injury (SCI). Nerve transfers and tendon transfers present several advantages and limitations, which must be discussed with the surgical candidate, based on their pattern of injury and functional requirements.

We propose a novel combined technique of tendon and nerve transfer to restore elbow extension by transferring the posterior deltoid tendon to the triceps tendon and the branch of teres minor nerve to the long head of the triceps nerve via the same posterior shoulder surgical approach. Since February 2018, we have treated three individuals with SCIs. Here we report the first patient's clinical course with 17 months of follow-up. As compared with isolated nerve or tendon transfer, this technique can reduce the failure rate

Marco Biondi

marcobiondi@ymail.com

1 Surgery and Reconstructive Microsurgery Unit of the Hand, Azienda Ospedaliero Universitaria Careggi, Largo Palagi 1, 50100 Florence, Italy

2 Department of Spinal Unit, Azienda Ospedaliero-Universitaria Careggi, Largo Palagi 1, 50100 Florence, Italy giving a more rapid, secure, and physiologic recovery of elbow extension in individuals with SCI.

\section{Case report}

A 21-year-old male developed C6, ASIA A tetraplegia after car injury (according to the International Standards for Neurological Classification of Spinal Cord Injury). He presented to our SCI clinic 8 months later, with no further spontaneous recovery of function expected. He was evaluated as group 5 on right side and group 3 on left side according to the International Classification for Surgery of the Hand in Tetraplegia. On left side, elbow extension was graded as M2, elbow flexion M5, shoulder adduction M5, and shoulder external rotation M5 according to the British Medical Research Council guidelines. Electromyography was performed preoperatively, demonstrating that the triceps was not completely denervated, although some fibrillations were observed, revealing a component of lower motor neuron injury in addition to the expected upper motor neuron injury. At 14 months after injury, the individual underwent elbow extension reconstruction via tendon and nerve transfer, plus transfer of the supinator nerve branches to the posterior interosseous nerve for finger and thumb extension. The local ethics committee approved the protocol and the patient signed informed consent before participation. 


\section{Surgical technique}

The patient was placed in prone position under general anesthesia without muscle relaxants to allow intraoperative nerve stimulation. A skin incision was outlined from $3 \mathrm{~cm}$ under the lateral border of the scapular spine along the posterior border of the deltoid and stopped just distal to deltoid tuberosity.

The first step was to retract the posterior border of the deltoid laterally in order to expose the teres minor muscle and the axillary nerve that lie under the deltoid muscle. The axillary nerve was followed into the quadrangular space to identify the nerve branch of teres minor muscle. The motor nerve has a cranial direction and can be easily followed distally on the posterior surface of the muscle until its divisions. Intraoperative electrical stimulation confirmed the identity and functional activity of the teres minor nerve branch. The next step was to identify the radial nerve on the triangular space, below the teres major muscle and anterior to the long head of the triceps muscle, for which medial retraction allows a wider exposure of the space. The nerve branch to the long head of the triceps was identified slightly medial to the radial nerve. Electrical stimulation of the triceps long head motor branch confirmed its identity and contraction. A small incision of $3 \mathrm{~cm}$ was performed on the inferior border of the teres major muscle to dissect proximally the nerve, ensuring adequate redundancy for direct end-to-end suture. The teres minor motor branch was freed and completely sectioned distally and sutured to the triceps long head motor branch using a tension-free 9-0 nylon suture. By the same surgical approach, the posterior deltoid tendon was transferred to the triceps tendon with interposition of a fascia lata allograft (Fig. 1). Transfer of the two nerve branches to the supinator muscle to the posterior interosseous nerve was performed via anterior approach on the proximal third of the left forearm.

\section{Postoperative course}

Postoperatively, the elbow and the wrist were immobilized in full extension to protect both nerve and tendon transfers. After 15 days, the individual started physiotherapy sessions with early mobilization of the elbow and wrist. He wore a hinged brace of the elbow with flexion increasing $15^{\circ}$ per week. Normal activities were encouraged 8 weeks after surgery. During the postoperative period, we did not detect any disadvantages from sacrificing the teres minor motor branch, and patient detected no weakness of external rotation. After 5 months postoperatively, the individual started to develop increased elbow extension strength, which we believe was obtained from the tendon transfer. After 8 months, he revealed increased strength during independent transfer from chair to bed and during wheelchair propulsion, where the recovering long head of the triceps is particularly involved. While, after 3 months, he was able to extend his forearm over the head holding a 2-kg weight for ten repetitions; after 17 months, he could hold 5-kg for ten repetitions. At last follow-up, he was also able to actively extend the elbow with a weight of $6.5 \mathrm{-kg}$ on horizontal plane for a single repetition (Fig. 2). Active thumb and fingers extension was recovered starting 7 months after surgery, and an M4 was reached.

\section{Discussion}

In patients affected by SCI, elbow extension is critical for overhead activities, weight shifting, independent transfers, and to perform self-care tasks such as eating [1, 2]. Restoration of elbow extension also greatly increases wheelchair propulsion and the workspace of the hand in space by $800 \%$ [3]. It also improves function of distal tendon transfers, such as the transfer of the brachioradialis tendon to flexor pollicis longus for reconstruction of key pinch.
Fig. 1 Combined technique of tendon and nerve transfer to restore elbow extension. a Posterior deltoid tendon to the triceps tendon with interposition of fascia lata and $\mathbf{b}$ transfer of the branch of teres minor nerve to the long head of the triceps nerve using the same posterior surgical approach. (d) deltoid muscle, $(\mathrm{t})$ triceps muscle, $(\mathrm{tm})$ teres minor muscle, (Axn) Axillary nerve, (tmnb) teres minor nerve branch, (lhtnb) long head of the triceps nerve branch.

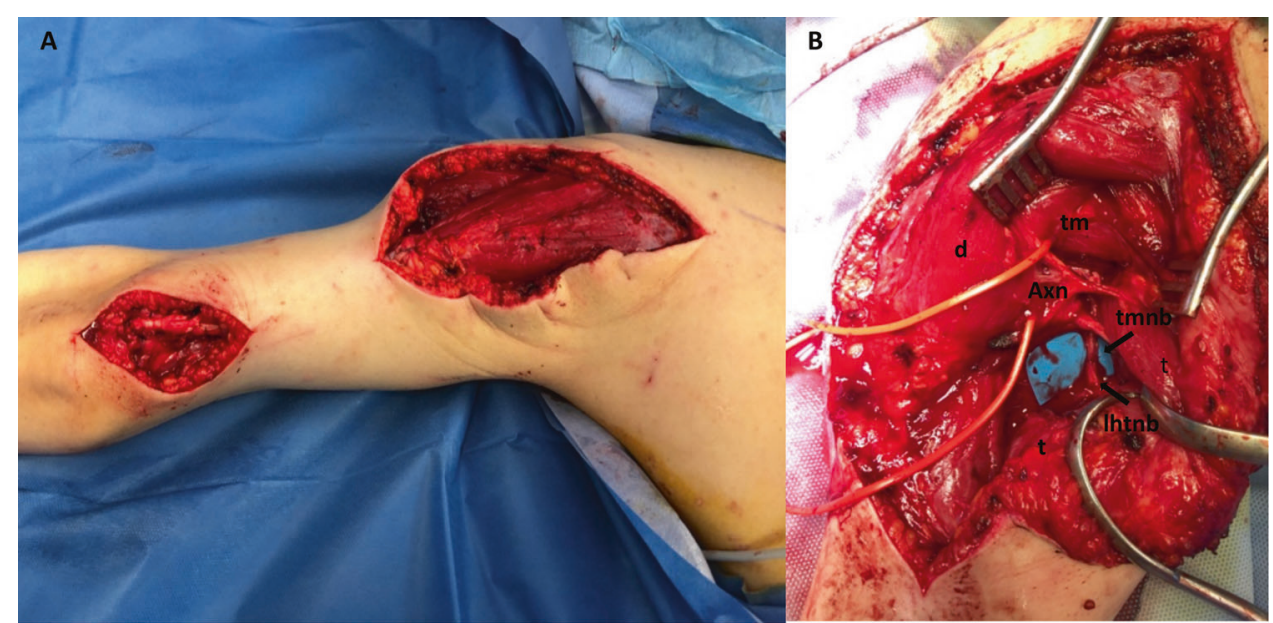




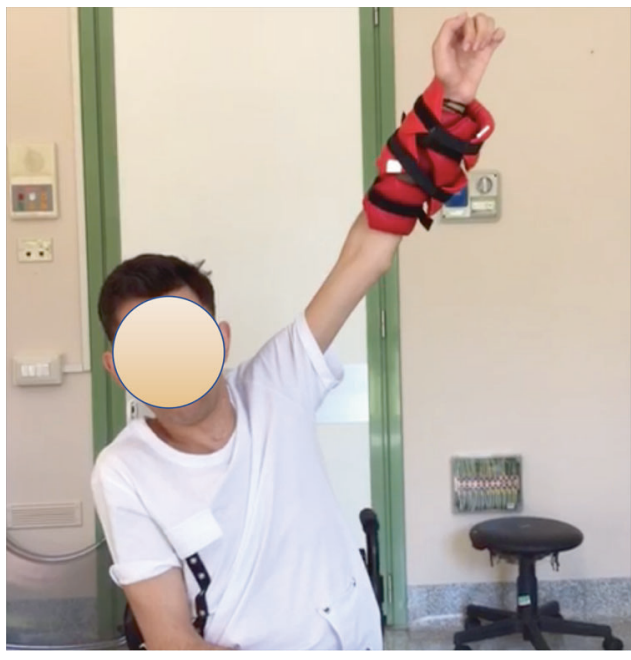

Fig. 2 Clinical Outcome. After 17 months the patient was able to extend his forearm over the head holding a $5-\mathrm{kg}$ weight for ten repetitions.

Two surgical tendon transfers are advocated to restore elbow extension: posterior deltoid-to-triceps transfer, and biceps-to-triceps tendon transfer. Which technique to use is based on the surgeon's preference and experience, though, for some patients, surgical prerequisites determine whether the biceps or the deltoid is transferred. Although the maximum force the biceps can generate is greater than that of the posterior deltoid [4], this procedure is not recommended when a supinator nerve branch is transferred to the posterior interosseous nerve or when the brachialis nerve is transferred to the median nerve [5]. For these reasons, the posterior deltoid tendon transfer is the preferred technique when a nerve transfer is planned to restore finger and wrist function. Hamou et al. [6] found that the mean Medical Research Council score for elbow extension after deltoid reconstruction was 3.3. The most common adverse events of elbow reconstruction with deltoid-to-triceps tendon are rupture or stretching of the graft.

Nerve transfers are a relative new procedure in SCI with encouraging preliminary functional results, although not yet established in a long term follow-up. These consist of crossing an expandable donor nerve, over which the patient has volitional control, to another nonfunctional recipient in order to restore volitional control of a muscle, which is anatomically and mechanically designed to perform that function. A single donor nerve can reinnervate multiple muscles, and restore multiple functions. When compared with peripheral nerve lesions, the most appropriate fascicles of the recipient nerve can be precisely determined by intraoperative nerve stimulation. When the lower motor neuron and spinal reflexes are intact, theoretically, potential for reinnervation remains for years [7]. In contrast to tendon transfers, technical challenges like joint positioning and tendon tensioning are avoided. Furthermore, long postoperative immobilizations are unnecessary and postoperative complications such as tendon adhesions, stretching, and rupture are avoided [8].

Bertelli et al. [9] described an axillary approach using the teres minor branch as the donor nerve and the triceps long head branch as the recipient nerve to reconstruct elbow extension. Having promising results, he converted the technique, transferring the posterior deltoid branch of the axillary nerve to triceps branches of the radial nerve through the same axillary approach [10]. These procedures do not lead to loss of function because they use an expendable donor nerve, which is compensated for via a redundant muscle group or innervation. However, if a poor result will be achieved transferring the posterior division of the axillary nerve, another nerve transfer or the posterior deltoid tendon cannot be used and the biceps or lower trapezius tendons must be transferred.

Limitations of nerve transfers concern the difficulty to define surgical candidacy, the longtime needed to recover the expected functional gain, and the strength and quality of motor function recovered. It is also difficult to discern whether muscles are nonfunctional due to lack of cortical control or due to denervation secondary to lower motor neuron injury [11]. Although promising results, clinical outcomes are variable [12] and not yet predictable like tendon transfers. Clinical outcomes like M3 may bring an augmented tenodesis effect that may not satisfy the patient.

With this combined technique we obtained several advantages for the patient:

- Ensured a more secure function recovery and a better clinical outcome, adding the advantage of both techniques. - The failure rate of the combined procedures can be lower compared with each of them: tendon and nerve transfers restore elbow extension in two different ways not excluding themselves.

- Late complications, such as rupture or stretching of the transferred posterior deltoid tendon are reduced by triceps neurologic reanimation.

- Failure or partial nerve reanimation can be supported by posterior deltoid tendon transfer avoiding the need of challenging salvage procedures.

- Reaching an early elbow function due to tendon transfer is very important for patient satisfaction. The goals achieved can then improve over time thanks to triceps reinnervation.

- These procedures are performed by the same posterior approach: adding nerve transfer to deltoid-to-triceps tendon transfer is an easy and quick procedure.

- With this approach the coaptation of the nerve stumps is very close to the muscle entry point, minimizing delayed reinnervation, and consequently enhancing results. 
In conclusion, restoring elbow extension in patients with SCI can be performed with many surgical techniques. While tendon transfers present well established and predictable clinical outcomes, nerve transfers present encouraging preliminary results [13]. However, difficulty in defining the proper surgical candidate for a nerve transfer can bring poor clinical outcomes, especially in delayed surgeries. For this reason, a combined nerve and tendon transfer to restore elbow extension can be a good solution to reach the best reliable clinical outcome in terms of strength. A salvage surgery can be avoided if the nerve transfer fails. On the other hand, nerve transfer can reinnervate the triceps muscle, strengthening the posterior deltoid transfer and protecting from a stretching or rupture of the tendon. We think that this surgical technique can add the benefit of each tendon and nerve transfer leading to a reduction of failure rate with more predictable and good clinical outcomes.

\section{Compliance with ethical standards}

Conflict of interest The authors declare that they have no conflict of interest.

Publisher's note Springer Nature remains neutral with regard to jurisdictional claims in published maps and institutional affiliations.

\section{References}

1. Endress RD, Hentz VR. Biceps-to-triceps transfer technique. J Hand Surg Am. 2011;36:716-21.
2. Lamberg AS, Friden J. Changes in skills required for using a manual wheelchair after reconstructive hand surgery in tetraplegia. J Rehabil Med. 2011;43:714-9.

3. Hentz VR, Leclercq C. Surgical rehabilitation of the upper limb in tetraplegia. New York: W.B.Saunders; 2002. p. p97.

4. Muclahey MJ, Lutz C, Kozin SH, Betz RR. Prospective evaluation of biceps to triceps and deltoid to triceps or elbow extension in tetraplegia. J Hand Surg Am. 2003;28:964-71.

5. Hill EJR, Fox IK. Current best peripheral nerve transfer for Spinal Cord Injury. Plast Reconstr Surg. 2019;143:184e-8e.

6. Hamou C, Shah NR, DiPonio L, Curtin C. Pinch and elbow extension restoration in people with tetraplegia: a systematic review of the literature. J Hand Surg Am 1009;34:692-9.

7. Fridem J, Gohritz A. Novel concepts integrated in Neuromuscular assessments for surgical restoration of arm and hand function in tetraplegia. Phis Med Rehabil Clin N Am. 2012;23:33-50.

8. Van Zyl N, Hahn JB, Cooper CA, Weymouth MD, Flood SJ, Galea MP. Upper limb reinnervation in C6 tetraplegia using a triple nerve transfer: case report. J Hand Surg. 2014;39:1779-83.

9. Bertelli JA, Ghizoni MF, Tacca CP. Transfer of the teres minor motor branch for triceps reinnervation in tetraplegia. J Neurosurg. 2011;114:1457-60.

10. Bertelli JA, Ghizoni MF. Nerve transfers for elbow and finger extension reconstruction in midcervical spinal cord injuries. $\mathrm{J}$ Neurosurg. 2015;122:121-7.

11. Mandeville RM, Brown JM, Sheean GL. A neurophysiological approach to nerve transfer to restore upper limb function in cervical spinal cord injury. Neurodurg Focus. 2017;43:E6.

12. Bertelli JA, Ghizoni MF. Nerve transfers for restoration of finger flexion in patients with tetraplegia. J Neurosurg Spine. 2017;26:55-61.

13. Fox IK, Davidge KM, Novak CB, Hoben G, Kahn LC, Juknis N, et al. Nerve transfers to restore upper extremity function in cervical spinal cord injury: update and preliminary outcomes. Plast Reconstr Surg. 2015;136:780-92. 